\title{
Reachability and Controllability of Fractional Singular Dynamical Systems with Control Delay
}

\author{
Hai Zhang, ${ }^{1,2}$ Jinde Cao, ${ }^{1,3}$ and Wei Jiang ${ }^{4}$ \\ ${ }^{1}$ Department of Mathematics, Southeast University, Nanjing, Jiangsu 210096, China \\ ${ }^{2}$ Department of Mathematics, Anqing Normal University, Anqing, Anhui 246133, China \\ ${ }^{3}$ Department of Mathematics, King Abdulaziz University, Jeddah 21589, Saudi Arabia \\ ${ }^{4}$ School of Mathematical Sciences, Anhui University, Hefei, Anhui 230039, China \\ Correspondence should be addressed to Jinde Cao; jdcao@seu.edu.cn
}

Received 3 August 2013; Revised 25 September 2013; Accepted 25 September 2013

Academic Editor: Han H. Choi

Copyright (C) 2013 Hai Zhang et al. This is an open access article distributed under the Creative Commons Attribution License, which permits unrestricted use, distribution, and reproduction in any medium, provided the original work is properly cited.

\begin{abstract}
This paper is concerned with the reachability and controllability of fractional singular dynamical systems with control delay. The factors of such systems including the Caputo's fractional derivative, control delay, and singular coefficient matrix are taken into account synchronously. The state structure of fractional singular dynamical systems with control delay is characterized by analysing the state response and reachable set. A set of sufficient and necessary conditions of controllability for such systems are established based on the algebraic approach. Moreover, an example is provided to illustrate the effectiveness and applicability of the proposed criteria.
\end{abstract}

\section{Introduction}

Singular systems play important roles in mathematical modeling of real-life problems arising in a wide range of applications. Depending on the area of application, these models are also called descriptor systems, semistate systems, differentialalgebraic systems, or generalized state-space systems. In the past few decades, singular systems with integer-order derivative have been extensively studied due to the fact that singular systems describe practical systems better than normal ones (see [1-8] and references therein).

Fractional calculus and its applications to the sciences and engineering is a recent focus of interest to many researchers. Fractional differential equations have been proved to be an excellent tool in the modelling of many phenomena in various fields of engineering, physics, and economics. Actually, fractional differential equations are considered as alternative model to integer differential equations. Many practical systems can be represented more accurately through fractional derivative formulation. For more details on fractional calculus theory, one can see the monographs of Miller and Ross [9], Podlubny [10], Diethelm [11], and Kilbas et al. [12]. Fractional differential equations involving the Riemann-Liouville fractional derivative or the Caputo fractional derivative have been paid more and more attention in [13-18] and references therein.

On the other hand, it is well-known that the issue of controllability plays an important role in engineering and control theory, which has close connections to pole assignment, structural decomposition, quadratic optimal control, and controller design, and so on. The problem of controllability for various kinds of dynamical systems with integer derivative has been extensively studied [1, 3-5, 19-22]. In particular, Dai [1] has established the theory of integer-order linear singular control systems. Yip and Sincovec [3] and Tang and Li [4] have investigated the controllability and observability of integer-order singular dynamical systems. It is worth mentioning that notable contributions have been made to fractional control systems [23-35]. The different techniques have been applied to investigate the controllability of various fractional dynamical systems, such as algebraic method [23, 24], geometrical analysis [25], fixed point theorems [26-30], and semigroup theory [31]. Recently, Sakthivel et al. [3235] have investigated the approximate controllability problem for various kinds of fractional dynamical systems by using fixed point techniques, which have further enriched and 
developed the control theory of fractional dynamical systems. The earlier studies concerning the controllability of fractional dynamical systems with control delay can be found in $[25,27$, 29 ], which especially focused on fractional normal systems. However, it should be emphasized that the control theory of fractional singular systems is not yet sufficiently elaborated, compared to that of fractional normal systems. In this regard, it is necessary and important to study the controllability problems for fractional singular dynamical systems. To the best of our knowledge, there are no relevant reports on reachability and controllability of fractional singular dynamical systems as treated in the current literature. Motivated by this consideration, in this paper, we investigate the reachability and controllability of fractional singular dynamical systems with control delay.

In this paper, we consider the reachability and controllability of the following fractional singular dynamical systems with control delay:

$$
\begin{gathered}
E^{c} D^{\alpha} x(t)=A x(t)+B u(t)+C u(t-\tau), \quad t \geq 0, \\
u(t)=\psi(t), \quad-\tau \leq t \leq 0, \\
x(0)=x_{0},
\end{gathered}
$$

where ${ }^{c} D^{\alpha} x(t)$ denotes an $\alpha$ order Caputo fractional derivative of $x(t)$, and $0<\alpha \leq 1 ; E, A, B$, and $C$ are the known constant matrices, $E, A \in \mathbb{R}^{n \times n}, B \in \mathbb{R}^{n \times m}, C \in \mathbb{R}^{n \times m}$, and $\operatorname{rank}(E)<n ; x \in \mathbb{R}^{n}$ is the state variable; $u \in \mathbb{R}^{m}$ is the control input; $\tau>0$ is the time control delay; and $\psi(t)$ is the initial control function.

The main purpose of this paper is to establish reachability and controllability criteria for system (1) based on the algebraic approach. The factors of fractional singular dynamical systems including the Caputo's fractional derivative, control delay, and singular coefficient matrices are taken into account synchronously. As discussed by Dai [1], we transform system (1) into two subsystems by the first equivalent form (FE1), which is more convenient to characterize the state reachable set. The state response, reachable set, and sufficient and necessary conditions for the controllability are obtained, respectively. The proposed criteria are applicable to a larger class of fractional dynamical systems. Therefore, we extend the known results $[1,23,25]$ to a more general case.

This paper is organized as follows. In the next section, we recall some definitions and preliminary facts used in the paper. In Section 3, the state structure of system (1) is characterized by analysing the state response and reachable set. In Section 4, the necessary and sufficient conditions of controllability for two subsystems and system (1) are derived, respectively. In Section 5, an example is provided to illustrate the effectiveness and applicability of the proposed criteria. Finally, some concluding remarks are drawn in Section 6.

\section{Preliminaries}

In this section, we first recall some definitions of fractional calculus and preliminary facts. For more details, one can see [9-12]. Next, the first equivalent form (FE1) of system (1) is given. (a) The Riemann-Liouville's fractional integral of order $\alpha>0$ with the lower limit zero for a function $f: R^{+} \rightarrow R^{n}$ is defined as

$$
D^{-\alpha} f(t)=\frac{1}{\Gamma(\alpha)} \int_{0}^{t}(t-s)^{\alpha-1} f(s) d s, \quad t>0,
$$

provided that the right side is pointwise defined on $[0,+\infty)$, where $\Gamma(\cdot)$ is the Gamma function.

(b) The Caputo's fractional derivative of order $\alpha$ for a function $f: R^{+} \rightarrow R^{n}$ is defined as

$$
\begin{array}{r}
{ }^{c} D^{\alpha} f(t)=\frac{1}{\Gamma(m-\alpha+1)} \int_{0}^{t}(t-s)^{m-\alpha} f^{(m+1)}(s) d s, \\
0 \leq m \leq \alpha<m+1 .
\end{array}
$$

For $0<\alpha \leq 1$,

$$
D^{-\alpha}\left\{{ }^{c} D^{\alpha} f(t)\right\}=f(t)-f(0) .
$$

(c) The Mittag-Leffler function in two parameters is defined as

$$
E_{\alpha, \beta}(z)=\sum_{k=0}^{+\infty} \frac{z^{k}}{\Gamma(k \alpha+\beta)}
$$

where $\alpha>0, \beta>0$, and $z \in \mathbb{C}, \mathbb{C}$ denotes the complex plane. In particular, for $\beta=1$,

$$
E_{\alpha, 1}(z)=E_{\alpha}(z)=\sum_{k=0}^{+\infty} \frac{z^{k}}{\Gamma(k \alpha+1)}
$$

has the interesting property

$$
{ }^{c} D^{\alpha} E_{\alpha}\left(\lambda z^{\alpha}\right)=\lambda E_{\alpha}\left(\lambda z^{\alpha}\right), \quad \lambda, z \in \mathbb{C} .
$$

(d) The Laplace transform of a function $f(t)$ is defined as

$$
F(s)=£[f(t)]=\int_{0}^{+\infty} e^{-s t} f(t) d t, \quad s \in \mathbb{C},
$$

where $f(t)$ is $n$-dimensional vector-valued function. For $\alpha \epsilon$ $(0,1]$, it follows from $[10]$ that

$$
\mathfrak{E}\left[\left({ }^{c} D^{\alpha} f\right)(t)\right]=s^{\alpha} \mathfrak{E}[f(t)]-s^{\alpha-1} f(0) .
$$

(e) For $n \in \mathbb{N}$, the sequential fractional derivative for suitable function $y(x)$ is defined by

$$
y^{(k)}:=\left(\mathbf{D}^{k \alpha} y\right)(x)=\left(\mathbf{D}^{\alpha} \mathbf{D}^{(k-1) \alpha} y\right)(x),
$$

where $k=1, \ldots, n$, and $\mathbf{D}^{\alpha}$ is any fractional differential operator; here we still mention it as ${ }^{c} D^{\alpha}$.

Now, we introduce the first equivalent form (FE1) of system (1) by the nonsingular transform, which is also called the standard decomposition of a singular system. 
Assume that $(E, A)$ is regular throughout this paper. From $[1,3]$, there exist two nonsingular matrices $P, Q \in \mathbb{R}^{n \times n}$, such that system (1) is equivalent to canonical system as follows:

$$
\begin{gathered}
{ }^{c} D^{\alpha} x_{1}(t)=A_{1} x_{1}(t)+B_{1} u(t)+C_{1} u(t-\tau), \quad t \geq 0, \\
N^{c} D^{\alpha} x_{2}(t)=x_{2}(t)+B_{2} u(t)+C_{2} u(t-\tau), \quad t \geq 0, \\
u(t)=\psi(t), \quad-\tau \leq t \leq 0, \\
x_{1}(0)=x_{10}, \quad x_{2}(0)=x_{20},
\end{gathered}
$$

where $x_{1} \in \mathbb{R}^{n_{1}}, x_{2} \in \mathbb{R}^{n_{2}}, n_{1}+n_{2}=n$, and $N$ is nilpotent whose nilpotent index is denoted by $v$; that is, $N^{v}=0$, $N^{\nu-1} \neq 0$, and

$$
\begin{gathered}
P E Q=\left[\begin{array}{cc}
I_{n_{1}} & 0 \\
0 & N
\end{array}\right], \quad P A Q=\left[\begin{array}{cc}
A_{1} & 0 \\
0 & I_{n_{2}}
\end{array}\right], \\
P B=\left[\begin{array}{l}
B_{1} \\
B_{2}
\end{array}\right], \quad P C=\left[\begin{array}{l}
C_{1} \\
C_{2}
\end{array}\right], \quad Q^{-1} x(t)=\left[\begin{array}{l}
x_{1}(t) \\
x_{2}(t)
\end{array}\right] .
\end{gathered}
$$

Let $h=[\alpha \nu]+1$, where $[\alpha \nu]$ denotes the integral part of $\alpha \nu$. Let $\mathbf{C}_{p}^{h}\left([0,+\infty), \mathbb{R}^{m}\right)$ be the set of $h$ times piecewise continuously differentiable functions mapping the interval $[0,+\infty)$ into $\mathbb{R}^{m}$. Moreover, $\mathbf{C}^{h}\left([-\tau, 0], \mathbb{R}^{m}\right)$ denotes the set of $h$ times continuously differentiable functions mapping the interval $[-\tau, 0]$ into $\mathbb{R}^{m}$.

Applying the method in [3], we can obtain the precise form of the admissible initial state set $S(\psi)$ for system (11). For $\psi \in \mathbf{C}^{h}\left([-\tau, 0], \mathbb{R}^{m}\right)$, we have

$$
\begin{aligned}
& S(\psi) \\
& :=\left\{x \in \mathbb{R}^{n} \mid x=\left[\begin{array}{l}
x_{1} \\
x_{2}
\end{array}\right], x_{1} \in \mathbb{R}^{n_{1}},\right. \\
& \left.\quad x_{2}=-\sum_{k=0}^{\nu-1}\left[N^{k} B_{2}{ }^{c} D^{k \alpha} \psi(0)+N^{k} C_{2}{ }^{c} D^{k \alpha} \psi(-\tau)\right]\right\} .
\end{aligned}
$$

Thus, the set of admissible initial data is

$$
\mathscr{A}:=\left\{\left(x_{0}, \psi\right) \mid \psi \in \mathbf{C}^{h}\left([-\tau, 0], \mathbb{R}^{m}\right), x_{0} \in S(\psi)\right\}
$$

\section{State Response and State Reachability}

In this section, we characterize the state structure of system (11) by analysing the state response and reachable set.
Theorem 1. For any admissible initial data $\left(x_{0}, \psi\right) \in \mathscr{A}$ and the control function $u(t) \in \mathbf{C}_{p}^{h}\left([0,+\infty), \mathbb{R}^{m}\right)$, the state response for system (11) can be represented in the following form:

$$
\begin{aligned}
x_{1}(t)= & E_{\alpha, 1}\left(A_{1} t^{\alpha}\right) x_{1}(0) \\
& +\int_{t-\tau}^{t}(t-s)^{\alpha-1} E_{\alpha, \alpha}\left[A_{1}(t-s)^{\alpha}\right] B_{1} u(s) d s \\
& +\int_{0}^{t-\tau}\left\{(t-s)^{\alpha-1} E_{\alpha, \alpha}\left[A_{1}(t-s)^{\alpha}\right] B_{1}+(t-\tau-s)^{\alpha-1}\right. \\
& \left.\times E_{\alpha, \alpha}\left[A_{1}(t-\tau-s)^{\alpha}\right] C_{1}\right\} u(s) d s \\
& +\int_{-\tau}^{0}(t-\tau-s)^{\alpha-1} E_{\alpha, \alpha}\left[A_{1}(t-\tau-s)^{\alpha}\right] C_{1} \psi(s) d s, \quad t \geq 0, \\
x_{2}(t)= & -\sum_{k=0}^{v-1}\left[N^{k} B_{2}{ }^{c} D^{k \alpha} u(t)+N^{k} C_{2}{ }^{c} D^{k \alpha} u(t-\tau)\right], \quad t \geq 0,
\end{aligned}
$$

where $E_{\alpha, \beta}(\cdot)$ is the Mittag-Leffler function and ${ }^{c} D^{k \alpha} u(\cdot)$ is the sequential fractional derivative.

Proof. From [10], the Laplace transform of the $\alpha$ order Caputo fractional derivative of function $x(t)$ is

$$
\mathfrak{E}\left[D^{\alpha} x(t)\right]=s^{\alpha} \mathfrak{E}[x(t)]-s^{\alpha-1} x(0) .
$$

Taking the Laplace transform with respect to $t$ in both sides of the first equation of system (11), we obtain

$$
\begin{aligned}
& s^{\alpha} £\left[x_{1}(t)\right]-s^{\alpha-1} x_{1}(0) \\
& \quad=A_{1} £\left[x_{1}(t)\right]+B_{1} £[u(t)]+C_{1} £[u(t-\tau)] .
\end{aligned}
$$

Hence,

$$
\begin{aligned}
\mathfrak{E}\left[x_{1}(t)\right]= & \left(s^{\alpha} I-A_{1}\right)^{-1} s^{\alpha-1} x_{1}(0) \\
& +\left(s^{\alpha} I-A_{1}\right)^{-1} \mathfrak{E}\left[B_{1} u(t)+C_{1} u(t-\tau)\right] .
\end{aligned}
$$

From [10], we have

$$
£\left[t^{\beta-1} E_{\alpha, \beta}\left(A_{1} t^{\alpha}\right)\right]=s^{\alpha-\beta}\left(s^{\alpha} I-A_{1}\right)^{-1} .
$$

Then (19) is equivalent to

$$
\begin{aligned}
£\left[x_{1}(t)\right]= & £\left[E_{\alpha, 1}\left(A_{1} t^{\alpha}\right)\right] x_{1}(0) \\
& +£\left[t^{\alpha-1} E_{\alpha, \alpha}\left(A_{1} t^{\alpha}\right)\right] £\left[B_{1} u(t)+C_{1} u(t-\tau)\right] .
\end{aligned}
$$

The convolution theorem of the Laplace transform applied to (21) yields the form

$$
\begin{aligned}
\mathfrak{E}\left[x_{1}(t)\right]= & £\left[E_{\alpha, 1}\left(A_{1} t^{\alpha}\right)\right] x_{1}(0) \\
+ & £\left\{\int_{0}^{t}(t-s)^{\alpha-1} E_{\alpha, \alpha}\left[A_{1}(t-s)^{\alpha}\right]\right. \\
& \left.\times\left[B_{1} u(s)+C_{1} u(s-\tau)\right] d s\right\} .
\end{aligned}
$$


Applying the inverse Laplace transform, we obtain

$$
\begin{aligned}
x_{1}(t)= & E_{\alpha, 1}\left(A_{1} t^{\alpha}\right) x_{1}(0) \\
& +\int_{0}^{t}(t-s)^{\alpha-1} E_{\alpha, \alpha}\left[A_{1}(t-s)^{\alpha}\right] \\
& \quad \times\left[B_{1} u(s)+C_{1} u(s-\tau)\right] d s \\
= & E_{\alpha, 1}\left(A_{1} t^{\alpha}\right) x_{1}(0) \\
& +\int_{t-\tau}^{t}(t-s)^{\alpha-1} E_{\alpha, \alpha}\left[A_{1}(t-s)^{\alpha}\right] B_{1} u(s) d s \\
& +\int_{0}^{t-\tau}\left\{(t-s)^{\alpha-1} E_{\alpha, \alpha}\left[A_{1}(t-s)^{\alpha}\right] B_{1}+(t-\tau-s)^{\alpha-1}\right. \\
& +\int_{-\tau}^{0}(t-\tau-s)^{\alpha-1} E_{\alpha, \alpha}\left[A_{1}(t-\tau-s)^{\alpha}\right] C_{1} \psi(s) d s .
\end{aligned}
$$

On the other hand, inserting (16) into both sides of the second equation in system (11) yields

$$
\begin{aligned}
& N^{c} D^{\alpha} x_{2}(t)-x_{2}(t)-B_{2} u(t)-C_{2} u(t-\tau) \\
& =-\sum_{k=0}^{\nu-1}\left[N^{k+1} B_{2}{ }^{c} D^{(k+1) \alpha} u(t)+N^{k+1} C_{2}{ }^{c} D^{(k+1) \alpha} u(t-\tau)\right] \\
& -B_{2} u(t)-C_{2} u(t-\tau) \\
& +\sum_{k=0}^{\nu-1}\left[N^{k} B_{2}{ }^{c} D^{k \alpha} u(t)+N^{k} C_{2}{ }^{c} D^{k \alpha} u(t-\tau)\right] \\
& =0 \text {. }
\end{aligned}
$$

Then (16) is a solution of the second equation in system (11).

Next, we will show that any solution of the second equation in system (11) has the form of (16). Define linear operator $T: \mathbf{C}^{1}\left([0,+\infty), \mathbb{R}^{n_{2}}\right) \rightarrow \mathbf{C}\left([0,+\infty), \mathbb{R}^{n_{2}}\right)$ by $T x_{2}(t)={ }^{c} D^{\alpha} x_{2}(t)$. Then the second equation in system (11) becomes

$$
(I-N T) x_{2}(t)=-B_{2} u(t)-C_{2} u(t-\tau) .
$$

Since $N$ is nilpotent, $N$ and $T$ can exchange, and ( $I$ $N T)\left(\sum_{k=0}^{v-1} N^{k} T^{k}\right)=I$, then we have

$$
\begin{aligned}
x_{2}(t) & =-(I-N T)^{-1}\left[B_{2} u(t)+C_{2} u(t-\tau)\right] \\
& =-\left(\sum_{k=0}^{+\infty} N^{k} T^{k}\right)\left[B_{2} u(t)+C_{2} u(t-\tau)\right] \\
& =-\left(\sum_{k=0}^{\nu-1} N^{k} T^{k}\right)\left[B_{2} u(t)+C_{2} u(t-\tau)\right] \\
& =-\sum_{k=0}^{\nu-1}\left[N^{k} B_{2}{ }^{c} D^{k \alpha} u(t)+N^{k} C_{2}{ }^{c} D^{k \alpha} u(t-\tau)\right] .
\end{aligned}
$$

Thus, (16) is indeed a solution of the second equation in system (11).

Therefore, the proof of Theorem 1 is completed.

For convenience sake, some notations are introduced as follows.

Given matrices $A \in \mathbb{R}^{n \times n}$ and $B, C \in \mathbb{R}^{n \times m}$, denote $\operatorname{Im}(B)$ as the range of $B$; that is,

$$
\operatorname{Im}(B)=\left\{y \mid y=B x, \forall x \in \mathbb{R}^{m}\right\} .
$$

Let

$$
\begin{aligned}
\langle A \mid B, C\rangle= & \operatorname{Im}(B)+\operatorname{Im}(A B)+\cdots+\operatorname{Im}\left(A^{n-1} B\right) \\
& +\operatorname{Im}(C)+\operatorname{Im}(A C)+\cdots+\operatorname{Im}\left(A^{n-1} C\right) .
\end{aligned}
$$

Then the space $\left\langle A_{1} \mid B_{1}, C_{1}\right\rangle$ is spanned by the columns of

$$
\left[B_{1}, A_{1} B_{1}, \ldots, A_{1}^{n_{1}-1} B_{1}, C_{1}, A_{1} C_{1}, \ldots, A_{1}^{n_{1}-1} C_{1}\right],
$$

and the space $\left\langle N \mid B_{2}, C_{2}\right\rangle$ is spanned by the columns of

$$
\left[B_{2}, N B_{2}, \ldots, N^{n_{2}-1} B_{2}, C_{2}, N C_{2}, \ldots, N^{n_{2}-1} C_{2}\right] .
$$

For any nonzero polynomial $f(s)$, we define $W(f, t): \mathbb{R}^{n_{1}} \rightarrow$ $\mathbb{R}^{n_{1}}$ by

$$
\begin{aligned}
& W(f, t) z \\
& =\int_{t-\tau}^{t} f(s)(t-s)^{\alpha-1} E_{\alpha, \alpha}\left[A_{1}(t-s)^{\alpha}\right] \\
& \quad \times B_{1} B_{1}^{*}(t-s)^{\alpha-1} E_{\alpha, \alpha}\left[A_{1}^{*}(t-s)^{\alpha}\right] f(s) z d s \\
& +\int_{0}^{t-\tau} f(s)\left\{(t-s)^{\alpha-1} E_{\alpha, \alpha}\left[A_{1}(t-s)^{\alpha}\right] B_{1}\right. \\
& \left.\quad+(t-\tau-s)^{\alpha-1} E_{\alpha, \alpha}\left[A_{1}(t-\tau-s)^{\alpha}\right] C_{1}\right\} \\
& \quad \times\left\{C_{1}^{*}(t-\tau-s)^{\alpha-1} E_{\alpha, \alpha}\left[A_{1}^{*}(t-\tau-s)^{\alpha}\right]\right. \\
& \left.+B_{1}^{*}(t-s)^{\alpha-1} E_{\alpha, \alpha}\left[A_{1}^{*}(t-s)^{\alpha}\right] f(s)\right\} z d s,
\end{aligned}
$$

where $*$ denotes the matrix transpose.

The following lemma is required for the main result, which is a natural extension of Lemma 2-1.1 in [1].

Lemma 2. Given matrices $A_{1} \in \mathbb{R}^{n_{1} \times n_{1}}$ and $B_{1}, C_{1} \in \mathbb{R}^{n_{1} \times m_{1}}$, then

$$
\operatorname{Im} W(f, t)=\left\langle A_{1} \mid B_{1}, C_{1}\right\rangle
$$

Proof. The formula (32) holds if and only if

$$
\operatorname{Ker} W(f, t)=\bigcap_{i=0}^{n_{1}-1} \operatorname{Ker} B_{1}^{*}\left(A_{1}^{*}\right)^{i} \bigcap_{i=0}^{n_{1}-1} \operatorname{Ker} C_{1}^{*}\left(A_{1}^{*}\right)^{i} .
$$


If $z_{0} \in \operatorname{Ker} W(f, t)$ and $z_{0} \neq 0, z_{0}^{*} G(t) z_{0}=0$, that is,

$$
\begin{gathered}
\int_{t-\tau}^{t} \| B_{1}^{*}(t-s)^{\alpha-1} E_{\alpha, \alpha}\left[\left(A_{1}^{*}(t-s)\right] f(s) z_{0} \|^{2} d s\right. \\
+\int_{0}^{t-\tau} \|\left[B_{1}^{*}(t-s)^{\alpha-1} E_{\alpha, \alpha}\left(A_{1}^{*}(t-s)\right)+C_{1}^{*}(t-\tau-s)^{\alpha-1}\right. \\
\left.\times E_{\alpha, \alpha}\left(A_{1}^{*}(t-\tau-s)\right)\right] f(s) z_{0} \|^{2} d s=0
\end{gathered}
$$

then we have

$$
\begin{aligned}
& B_{1}^{*}(t-s)^{\alpha-1} E_{\alpha, \alpha}\left[A_{1}^{*}(t-s)\right] f(s) z_{0}=0, \quad s \in[t-\tau, t], \\
& {\left[B_{1}^{*}(t-s)^{\alpha-1} E_{\alpha, \alpha}\left(A_{1}^{*}(t-s)\right)+C_{1}^{*}(t-\tau-s)^{\alpha-1}\right.} \\
& \left.\quad \times E_{\alpha, \alpha}\left(A_{1}^{*}(t-\tau-s)\right)\right] f(s) z_{0}=0, \quad s \in[0, t-\tau] .
\end{aligned}
$$

Since polynomial $f(s)$ only has a finite number of zero on $t-\tau \leq s \leq t$ and $0 \leq s \leq t-\tau$, we immediately have

$$
\begin{gathered}
B_{1}^{*}(t-s)^{\alpha-1} E_{\alpha, \alpha}\left[A_{1}^{*}(t-s)\right] z_{0}=0, \quad s \in[t-\tau, t], \\
{\left[B_{1}^{*}(t-s)^{\alpha-1} E_{\alpha, \alpha}\left(A_{1}^{*}(t-s)\right)+C_{1}^{*}(t-\tau-s)^{\alpha-1}\right.} \\
\left.\quad \times E_{\alpha, \alpha}\left(A_{1}^{*}(t-\tau-s)\right)\right] z_{0}=0, \quad s \in[0, t-\tau] .
\end{gathered}
$$

Repeatedly taking the Caputo's derivative on both sides of (37), we have

$$
\begin{array}{r}
B_{1}^{*}(t-s)^{\alpha-1} E_{\alpha, \alpha}\left[A_{1}^{*}(t-s)\right]\left(A_{1}^{*}\right)^{i} z_{0}=0, \\
i=1,2, \ldots, n_{1}-1, \quad s \in[t-\tau, t] .
\end{array}
$$

Substituting $s=t$ in (39) yields

$$
B^{*}\left(A^{*}\right)^{i} z_{0}=0, \quad i=1,2, \ldots, n_{1}-1,
$$

which implies that $z_{0} \in \operatorname{Ker} B_{1}^{*}\left(A_{1}^{*}\right)^{i}, i=1,2, \ldots, n_{1}-1$. According to Cayley-Hamilton theorem [4], $(t-$ $s)^{\alpha-1} E_{\alpha, \alpha}\left[A_{1}(t-s)^{\alpha}\right]$ can be represented as

$$
\begin{aligned}
(t-s)^{\alpha-1} E_{\alpha, \alpha}\left[A_{1}(t-s)^{\alpha}\right] & =\sum_{k=0}^{+\infty} \frac{(t-s)^{k \alpha+\alpha-1}}{\Gamma(k \alpha+\alpha)} A_{1}^{k} \\
& =\sum_{k=0}^{n_{1}-1} \gamma_{k}(t-s) A_{1}^{k} .
\end{aligned}
$$

For $s \in[0, t-\tau]$, it follows from (39) and (41) that

$$
\begin{aligned}
& B_{1}^{*}(t-s)^{\alpha-1} E_{\alpha, \alpha}\left(A_{1}^{*}(t-s)\right) z_{0} \\
& \quad=\sum_{k=0}^{n_{1}-1} \gamma_{k}(t-s) B_{1}^{*} A_{1}^{* k} z_{0}=0 .
\end{aligned}
$$

Inserting (42) into (38) yields

$$
C_{1}^{*}(t-\tau-s)^{\alpha-1} E_{\alpha, \alpha}\left(A_{1}^{*}(t-\tau-s)\right) z_{0}=0 .
$$

Repeatedly taking the Caputo's fractional derivative on both sides of (43) and letting $s=t-\tau$, we have

$$
C_{1}^{*} A_{1}^{* k} z_{0}=0, \quad k=1,2, \ldots, n_{1}-1,
$$

which implies that $z_{0} \in \operatorname{Ker} C_{1}^{*}\left(A_{1}^{*}\right)^{i}, i=1,2, \ldots, n_{1}-1$. Therefore, we have

$$
\operatorname{Ker} W(f, t) \subseteq \bigcap_{i=0}^{n_{1}-1} \operatorname{Ker} B_{1}^{*}\left(A_{1}^{*}\right)^{i} \bigcap_{i=0}^{n_{1}-1} \operatorname{Ker} C_{1}^{*}\left(A_{1}^{*}\right)^{i} .
$$

Conversely, if $z_{0} \in \bigcap_{i=0}^{n_{1}-1} \operatorname{Ker} B_{1}^{*}\left(A_{1}^{*}\right)^{i} \bigcap_{i=0}^{n_{1}-1} \operatorname{Ker} C_{1}^{*}\left(A_{1}^{*}\right)^{i}$ and $z_{0} \neq 0$, then $z_{0} \in \operatorname{Ker} B_{1}^{*}\left(A_{1}^{*}\right)^{i}, z_{0} \in \operatorname{Ker} C_{1}^{*}\left(A_{1}^{*}\right)^{i}, i=$ $1,2, \ldots, n_{1}-1$, and

$$
B_{1}^{*}\left(A_{1}^{*}\right)^{i} z_{0}=C_{1}^{*}\left(A_{1}^{*}\right)^{i} z_{0}=0, \quad i=1,2, \ldots, n_{1}-1 .
$$

For $s \in[t-\tau, t]$, it follows from (41) that

$$
\begin{aligned}
B_{1}^{*}(t-s)^{\alpha-1} E_{\alpha, \alpha}\left(A_{1}^{*}(t-s)\right) z_{0} \\
=\sum_{k=0}^{n_{1}-1} \gamma_{k}(t-s) B_{1}^{*} A_{1}^{* k} z_{0}=0 .
\end{aligned}
$$

For $s \in[0, t-\tau]$, the same argument yields

$$
\begin{aligned}
& {\left[B_{1}^{*}(t-s)^{\alpha-1} E_{\alpha, \alpha}\left(A_{1}^{*}(t-s)\right)+C_{1}^{*}(t-\tau-s)^{\alpha-1}\right.} \\
& \left.\quad \times E_{\alpha, \alpha}\left(A_{1}^{*}(t-\tau-s)\right)\right] z_{0} \\
& =\sum_{k=0}^{n_{1}-1} \gamma_{k}(t-s) B_{1}^{*} A_{1}^{* k} z_{0} \\
& \quad+\sum_{k=0}^{n_{1}-1} \lambda_{k}(t-\tau-s) C_{1}^{*} A_{1}^{* k} z_{0}=0 .
\end{aligned}
$$

Hence, $z_{0} \in \operatorname{Ker} W(f, t)$, which implies

$$
\operatorname{Ker} W(f, t) \supseteq \bigcap_{i=0}^{n_{1}-1} \operatorname{Ker} B_{1}^{*}\left(A_{1}^{*}\right)^{i} \bigcap_{i=0}^{n_{1}-1} \operatorname{Ker} C_{1}^{*}\left(A_{1}^{*}\right)^{i} .
$$

From (45) and (49), we know that (32) is true. The proof is therefore completed.

The reachable set for system (1) (or (11)) may be defined as follows.

Definition 3. Any vector $\omega \in \mathbb{R}^{n}$ in $n$-dimensional vector space is said to reachable if there exists an admissible initial data $\left(x_{0}, \psi\right) \in \mathscr{A}$, admissible control input $u(t) \in$ $\mathrm{C}_{p}^{h}\left([0,+\infty), \mathbb{R}^{m}\right)$, and $t_{f}>0$ such that the solution of system (1) (or (11)) satisfies $x\left(t_{f}, x_{0}, \psi\right)=\omega$.

Let $\mathfrak{R}\left(x_{0}, \psi\right)$ be the reachable set from any admissible initial data $\left(x_{0}, \psi\right) \in \mathscr{A}$. Then

$$
\begin{aligned}
& \mathfrak{R}\left(x_{0}, \psi\right) \\
& =\left\{\omega \in \mathbb{R}^{n} \mid\left(x_{0}, \psi\right) \in \mathscr{A}, u(t) \in \mathbf{C}_{p}^{h}\left([0,+\infty), \mathbb{R}^{m}\right),\right. \\
& \left.\quad t_{f}>0, x\left(t_{f}, x_{0}, \psi\right)=\omega\right\} .
\end{aligned}
$$


Considering the reachable set from the initial conditions $x_{0}=0$ and $\psi \equiv 0$, we have the following theorem.

Theorem 4. For system (11), the reachable set $\mathfrak{R}(0,0)$ can be represented as

$$
\mathfrak{R}(0,0)=\left\langle A_{1} \mid B_{1}, C_{1}\right\rangle \oplus\left\langle N \mid B_{2}, C_{2}\right\rangle,
$$

where " $\oplus$ " is the direct sum in vector space.

Proof. Let $\omega \in \mathfrak{R}(0,0)$, from (15), (16), and (50); then there exists $t_{f}>0$ and $u(t) \in \mathbf{C}_{p}^{h}\left([0,+\infty), \mathbb{R}^{m}\right)$ such that

$$
\begin{aligned}
& \omega_{1}=\int_{0}^{t_{f}}\left(t_{f}-s\right)^{\alpha-1} E_{\alpha, \alpha}\left[A_{1}\left(t_{f}-s\right)^{\alpha}\right] B_{1} u(s) d s \\
& +\int_{0}^{t_{f}-\tau}\left(t_{f}-\tau-s\right)^{\alpha-1} E_{\alpha, \alpha}\left[A_{1}\left(t_{f}-\tau-s\right)^{\alpha}\right] \\
& \quad \times C_{1} u(s) d s .
\end{aligned}
$$

Combining (41) and (52) yields

$$
\begin{aligned}
\omega_{1}= & \sum_{k=0}^{n_{1}-1} \int_{0}^{t_{f}} \varphi_{k}\left(t_{f}-s\right) A_{1}^{k} B_{1} u(s) d s \\
& +\sum_{k=0}^{n_{1}-1} \int_{0}^{t_{f}-\tau} \phi_{k}\left(t_{f}-\tau-s\right) A_{1}^{k} C_{1} u(s) d s .
\end{aligned}
$$

Therefore, $\omega_{1} \in\left\langle A_{1} \mid B_{1}, C_{1}\right\rangle$. Moreover, we also have

$$
\omega_{2}=-\sum_{k=0}^{\nu-1}\left[N^{k} B_{2}{ }^{c} D^{k \alpha} u\left(t_{f}\right)+N^{k} C_{2}{ }^{c} D^{k \alpha} u\left(t_{f}-\tau\right)\right],
$$

which implies that $\omega_{2} \in\left\langle N \mid B_{2}, C_{2}\right\rangle$. Thus,

$$
\Re(0,0) \subseteq\left\langle A_{1} \mid B_{1}, C_{1}\right\rangle \oplus\left\langle N \mid B_{2}, C_{2}\right\rangle .
$$

On the other hand, we need to prove

$$
\Re(0,0) \supseteq\left\langle A_{1} \mid B_{1}, C_{1}\right\rangle \oplus\left\langle N \mid B_{2}, C_{2}\right\rangle .
$$

Suppose that

$$
\omega=\left[\begin{array}{l}
\omega_{1} \\
\omega_{2}
\end{array}\right] \in\left\langle A_{1} \mid B_{1}, C_{1}\right\rangle \oplus\left\langle N \mid B_{2}, C_{2}\right\rangle
$$

where $\omega_{1} \in\left\langle A_{1} \mid B_{1}, C_{1}\right\rangle, \omega_{2} \in\left\langle N \mid B_{2}, C_{2}\right\rangle$, and $\omega_{1} \neq 0$, $\omega_{2} \neq 0$. For the initial conditions $x_{0}=0$ and $\psi(t) \equiv 0, t \in$ $[-\tau, 0]$, let $u(s)=u_{1}(s)+u_{2}(s)$; then we have

$$
\begin{aligned}
x_{1}(t)= & \int_{t-\tau}^{t}(t-s)^{\alpha-1} E_{\alpha, \alpha}\left[A_{1}(t-s)^{\alpha}\right] B_{1}\left[u_{1}(s)+u_{2}(s)\right] d s \\
& +\int_{0}^{t-\tau}(t-s)^{\alpha-1} E_{\alpha, \alpha}\left[A_{1}(t-s)^{\alpha}\right] \\
& \times B_{1}\left[u_{1}(s)+u_{2}(s)\right] d s \\
& +\int_{0}^{t-\tau}(t-\tau-s)^{\alpha-1} E_{\alpha, \alpha}\left[A_{1}(t-\tau-s)^{\alpha}\right] \\
x_{2}(t)= & -\sum_{k=0}^{\nu-1}\left[N^{k} B_{2}{ }^{c} D^{k \alpha} u_{1}(t)+N^{k} C_{2}{ }^{c} D^{k \alpha} u_{1}(t-\tau)\right] \\
& -\sum_{k=0}^{\nu-1}\left[N^{k} B_{2}{ }^{c} D^{k \alpha} u_{2}(t)+N^{k} C_{2}{ }^{c} D^{k \alpha} u_{2}(t-\tau)\right] .
\end{aligned}
$$

As discussed by Yip and Sincovec [3], we choose $u_{1}(s)=$ $f(s) y(s)$ to satisfy

$$
\begin{aligned}
& \int_{0}^{t-\tau}(t-s)^{\alpha-1} E_{\alpha, \alpha}\left[A_{1}(t-s)^{\alpha}\right] B_{1} f(s) g(s) d s \\
& \quad+\int_{\tau}^{t}(t-s)^{\alpha-1} E_{\alpha, \alpha}\left[A_{1}(t-s)^{\alpha}\right] C_{1} f(s-\tau) g(s-\tau) d s \\
& =\omega_{1}-\int_{0}^{t-\tau}(t-s)^{\alpha-1} E_{\alpha, \alpha}\left[A_{1}(t-s)^{\alpha}\right] B_{1} u_{2}(s) d s \\
& \quad-\int_{0}^{t-\tau}(t-\tau-s)^{\alpha-1} E_{\alpha, \alpha}\left[A_{1}(t-\tau-s)^{\alpha}\right] \\
& \quad \times C_{1} u_{2}(s) d s \equiv \widehat{\omega}_{1}, \\
& { }^{c} D^{k \alpha} u_{1}(t)={ }^{c} D^{k \alpha} u_{1}(t-\tau)=0, \quad k=0,1,2, \ldots, v-1 .
\end{aligned}
$$

Then $u_{1}(s)$ contributes nothing to $x_{2}(t)$ at $s=t$ and $s=t-\tau$. Now, we prove that $(60)$ is true.

In fact, for $\widehat{\omega}_{1} \in\left\langle A_{1} \mid B_{1}, C_{1}\right\rangle$, from Lemma 2 , there exists $z \in \mathbb{R}^{n}$ such that $W(f, t) z=\widehat{\omega}_{1}$.

Let

$$
y(s)=\left\{\begin{array}{c}
f(s)\left[B_{1}^{*}(t-s)^{\alpha-1} E_{\alpha, \alpha}\left(A_{1}^{*}(t-s)\right)\right. \\
\left.+C_{1}^{*}(t-\tau-s)^{\alpha-1} E_{\alpha, \alpha}\left(A_{1}^{*}(t-\tau-s)\right)\right] z, 0 \leq s \leq t-\tau, \\
f(s) B_{1}^{*}(t-s)^{\alpha-1} E_{\alpha, \alpha}\left(A_{1}^{*}(t-s)\right) z \\
t-\tau \leq s \leq t,-\tau \leq s \leq 0 .
\end{array}\right.
$$


Then

$$
\begin{aligned}
& \int_{0}^{t-\tau}(t-s)^{\alpha-1} E_{\alpha, \alpha}\left[A_{1}(t-s)^{\alpha}\right] B_{1} u_{1}(s) d s \\
& +\int_{\tau}^{t}(t-s)^{\alpha-1} E_{\alpha, \alpha}\left[A_{1}(t-s)^{\alpha}\right] C_{1} u_{1}(s-\tau) d s \\
& =\int_{t-\tau}^{t} f(s)(t-s)^{\alpha-1} E_{\alpha, \alpha}\left[A_{1}(t-s)^{\alpha}\right] \\
& \quad \times B_{1} B_{1}^{*}(t-s)^{\alpha-1} E_{\alpha, \alpha}\left[A_{1}^{*}(t-s)^{\alpha}\right] f(s) z d s \\
& +\int_{0}^{t-\tau} f(s)\left\{(t-s)^{\alpha-1} E_{\alpha, \alpha}\left[A_{1}(t-s)^{\alpha}\right] B_{1}\right. \\
& \left.\quad+(t-\tau-s)^{\alpha-1} E_{\alpha, \alpha}\left[A_{1}(t-\tau-s)^{\alpha}\right] C_{1}\right\} \\
& \quad \times\left\{C_{1}^{*}(t-\tau-s)^{\alpha-1} E_{\alpha, \alpha}\left[A_{1}^{*}(t-\tau-s)^{\alpha}\right]\right. \\
& \left.\quad+B_{1}^{*}(t-s)^{\alpha-1} E_{\alpha, \alpha}\left[A_{1}^{*}(t-s)^{\alpha}\right] f(s)\right\} z d s \\
& =W(f, t) z=\widehat{\omega}_{1} .
\end{aligned}
$$

Therefore, (60) is true.

For $\omega_{2} \in\left\langle N \mid B_{2}, C_{2}\right\rangle$, there exist $y_{k}, z_{k}$ such that

$$
\omega_{2}=-\sum_{k=0}^{\nu-1} N^{k} B_{2} y_{k}-\sum_{k=0}^{\nu-1} N^{k} C_{2} z_{k}
$$

There exists a function $p(s)$ such that ${ }^{c} D^{k \alpha} p(0)=0$, ${ }^{c} D^{k \alpha} p(t)=y_{k}$, and ${ }^{c} D^{k \alpha} p(t-\tau)=z_{k}$. Let

$$
u_{2}(s)= \begin{cases}p(s), & 0 \leq s \leq t, \\ 0, & -\tau \leq s \leq 0 ;\end{cases}
$$

then we have

$$
\begin{aligned}
x_{2}(t)= & -\sum_{k=0}^{\nu-1} N^{k} B_{2}{ }^{c} D^{k \alpha} u_{2}(t) \\
& -\sum_{k=0}^{\nu-1} N^{k} C_{2}{ }^{c} D^{k \alpha} u_{2}(t-\tau)=\omega_{2},
\end{aligned}
$$

and $x_{2}(0)=0$. It follows from (60) and (65) that $\omega \in \mathfrak{R}(0,0)$. Thus, (56) holds. Combining (55) and (56), we know that (51) is true. Then the proof of Theorem 4 is completed.

\section{Controllability Criteria}

In this section, we proceed to investigate the controllability criteria of system (1) and system (11) based on the reachable set. A set of sufficient conditions and necessary conditions for the controllability are derived based on the algebraic approach.

Definition 5. System (1) (or (11)) is said to be controllable at $t_{f}>0$ if one can reach any state at $t_{f}$ from any admissible initial data $\left(x_{0}, \psi\right) \in \mathscr{A}$.
Theorem 6. Canonical system (11) is controllable if and only if

$$
\begin{aligned}
& \operatorname{rank}\left[B_{1}, A_{1} B_{1}, \ldots, A_{1}^{n_{1}-1} B_{1}, C_{1}, A_{1} C_{1}, \ldots, A_{1}^{n_{1}-1} C_{1}\right]=n_{1} \text {, } \\
& \operatorname{rank}\left[B_{2}, N B_{2}, \ldots, N^{n_{2}-1} B_{2}, C_{2}, N C_{2}, \ldots, N^{n_{2}-1} C_{2}\right]=n_{2} \text {. }
\end{aligned}
$$

Proof. We firstly prove the necessity of Theorem 6 . If system (11) is controllable, for any $\omega=\left[\begin{array}{c}\omega_{1} \\ \omega_{2}\end{array}\right] \in \mathbb{R}^{n}$, to the initial state $x_{0}=0$ and initial control function $\psi \equiv 0$, there exists $t_{f}>0$ and a control function $u(\cdot) \in \mathbf{C}_{p}^{h}\left([0,+\infty)\right.$ such that $\omega_{1}, \omega_{2}$ could be written in the form of (58) and (59). That is

$$
\omega \in\left\langle A_{1} \mid B_{1}, C_{1}\right\rangle \oplus\left\langle N \mid B_{2}, C_{2}\right\rangle,
$$

which implies that

$$
\mathbb{R}^{n} \subseteq\left\langle A_{1} \mid B_{1}, C_{1}\right\rangle \oplus\left\langle N \mid B_{2}, C_{2}\right\rangle .
$$

Obviously,

$$
\mathbb{R}^{n} \supseteq\left\langle A_{1} \mid B_{1}, C_{1}\right\rangle \oplus\left\langle N \mid B_{2}, C_{2}\right\rangle .
$$

Consequently, we have

$$
\begin{gathered}
\mathbb{R}^{n}=\left\langle A_{1} \mid B_{1}, C_{1}\right\rangle \oplus\left\langle N \mid B_{2}, C_{2}\right\rangle, \\
\left\langle A_{1} \mid B_{1}, C_{1}\right\rangle=\mathbb{R}^{n_{1}}, \quad\left\langle N \mid B_{2}, C_{2}\right\rangle=\mathbb{R}^{n_{2}} .
\end{gathered}
$$

Thus, (66) and (67) are true.

Next, we prove the sufficiency of Theorem 6. If (66) and (67) are true, then we know that (71) holds. For any $\omega \in \mathbb{R}^{n}$ and any initial state $x_{0}$ and initial control function $\psi(\cdot)$, let

$$
\begin{gathered}
k_{1}=\omega_{1}-E_{\alpha, 1}\left(A_{1} t^{\alpha}\right) x_{1}(0) \\
-\int_{t-\tau}^{t}(t-s)^{\alpha-1} E_{\alpha, \alpha}\left[A_{1}(t-s)^{\alpha}\right] B_{1} \psi(0) d s \\
-\int_{0}^{t-\tau}\left\{(t-s)^{\alpha-1} E_{\alpha, \alpha}\left[A_{1}(t-s)^{\alpha}\right] B_{1}+(t-\tau-s)^{\alpha-1}\right. \\
\left.\quad \times E_{\alpha, \alpha}\left[A_{1}(t-\tau-s)^{\alpha}\right] C_{1}\right\} \psi(0) d s \\
-\int_{-\tau}^{0}(t-\tau-s)^{\alpha-1} E_{\alpha, \alpha}\left[A_{1}(t-\tau-s)^{\alpha}\right] C_{1} \psi(s) d s, \\
k_{2}=\omega_{2}+B_{2} \psi(0)+C_{2} \psi(0) .
\end{gathered}
$$

For $k=\left[\begin{array}{l}k_{1} \\ k_{2}\end{array}\right] \in \mathbb{R}^{n}=\left\langle A_{1} \mid B_{1}, C_{1}\right\rangle \oplus\left\langle N \mid B_{2}, C_{2}\right\rangle$, we have $k \in \mathfrak{R}(0,0)$. Then there exists a control $\widetilde{u}(s)$ such that

$$
\begin{gathered}
k_{1}=\int_{t-\tau}^{t}(t-s)^{\alpha-1} E_{\alpha, \alpha}\left[A_{1}(t-s)^{\alpha}\right] B_{1} \widetilde{u}(s) d s \\
+\int_{0}^{t-\tau}\left[(t-s)^{\alpha-1} E_{\alpha, \alpha}\left[A_{1}(t-s)^{\alpha}\right] B_{1}+(t-\tau-s)^{\alpha-1}\right. \\
\left.\quad \times E_{\alpha, \alpha}\left[A_{1}(t-\tau-s)^{\alpha}\right] C_{1}\right] \widetilde{u}(s) d s, \\
k_{2}=-\sum_{k=0}^{\nu-1}\left[N^{k} B_{2}{ }^{c} D^{k \alpha} \widetilde{u}(t)+N^{k} C_{2}^{c} D^{k \alpha} \widetilde{u}(t-\tau)\right] .
\end{gathered}
$$


Let $u(s)=\tilde{u}(s)+\psi(0)$, then we have

$$
\begin{aligned}
\omega_{1}= & E_{\alpha, 1}\left(A_{1} t^{\alpha}\right) x_{1}(0) \\
& +\int_{t-\tau}^{t}(t-s)^{\alpha-1} E_{\alpha, \alpha}\left[A_{1}(t-s)^{\alpha}\right] B_{1} u(s) d s \\
& +\int_{0}^{t-\tau}\left\{(t-s)^{\alpha-1} E_{\alpha, \alpha}\left[A_{1}(t-s)^{\alpha}\right] B_{1}\right. \\
& \left.+(t-\tau-s)^{\alpha-1} E_{\alpha, \alpha}\left[A_{1}(t-\tau-s)^{\alpha}\right] C_{1}\right\} u(s) d s \\
& \int_{-\tau}^{0}(t-\tau-s)^{\alpha-1} E_{\alpha, \alpha}\left[A_{1}(t-\tau-s)^{\alpha}\right] C_{1} \psi(s) d s, \\
\omega_{2}= & -\sum_{k=0}^{v-1}\left[N^{k} B_{2}{ }^{c} D^{k \alpha} u(t)+N^{k} C_{2}{ }^{c} D^{k \alpha} u(t-\tau)\right] .
\end{aligned}
$$

Thus, by Definition 5, system (11) is controllable. Therefore, the proof is completed.

Applying the results of Yip and Sincovec [3], Tang and Li [4], and Theorem 6 to the pair of matrices, we can obtain the following results.

Theorem 7. Canonical system (11) is controllable if and only if

$$
\begin{gathered}
\operatorname{rank}\left[s I-A_{1}, B_{1}, C_{1}\right]=n_{1}, \quad \forall s \in \mathbb{C}, s \text { finite, } \\
\operatorname{rank}\left[N, B_{2}, C_{2}\right]=n_{2} .
\end{gathered}
$$

Theorem 8. System (1) is controllable if and only if

$$
\begin{gathered}
\operatorname{rank}[s E-A, B, C]=n, \quad \forall s \in \mathbb{C}, s \text { finite, } \\
\operatorname{rank}[E, B, C]=n .
\end{gathered}
$$

Remark 9. If we choose $\alpha=1$ and $C=0$, then system (1) reduces to an integer-order singular system without control delay. From Theorem 8, system (1) is controllable if and only if

$$
\begin{gathered}
\operatorname{rank}[s E-A, B]=n, \quad \forall s \in \mathbb{C}, s \text { finite, } \\
\operatorname{rank}[E, B]=n,
\end{gathered}
$$

which is just the result in [1].

If we choose $E=I, C=0$, then system (1) reduces to a fractional normal system without control delay. From Theorem 8, system (1) is controllable if and only if

$$
\operatorname{rank}\left[B, A B, \ldots, A^{n-1} B\right]=n,
$$

which is just Corollary 3.4 in [23].

If we choose $E=I$, then system (1) reduces to a fractional normal system with control delay. From Theorem 8, system (1) is controllable if and only if

$$
\operatorname{rank}\left[B, A B, \ldots, A^{n-1} B, C, A C, \ldots, A^{n-1} C\right]=n,
$$

which is just Theorem 3 in [25]. Therefore, our results extend the existing results $[1,23,25]$.
Remark 10. Theorem 8 actually offers an algebraic criterion of the exact controllability for linear fractional singular dynamical systems, which is concise and convenient to check the controllability of such systems. Motivated and inspired by the works of Sakthivel et al. [32-35], the approximate controllability of linear (nonlinear) fractional singular dynamical systems with the control delay will become our future investigative work.

\section{An Illustrative Example}

In this section, we give an example to illustrate the applications of Theorem 8 .

Example 11. Consider the controllability of fractional singular dynamical systems as follows:

$$
\begin{gathered}
{\left[\begin{array}{lll}
1 & 0 & 0 \\
0 & 0 & 1 \\
0 & 0 & 0
\end{array}\right] D^{1 / 2} x(t)=} \\
{\left[\begin{array}{lll}
1 & 0 & 0 \\
0 & 1 & 1 \\
1 & 0 & 1
\end{array}\right] x(t)+\left[\begin{array}{ll}
1 & 0 \\
1 & 1 \\
0 & 1
\end{array}\right] u(t)} \\
+\left[\begin{array}{ll}
1 & 0 \\
0 & 1 \\
1 & 1
\end{array}\right] u\left(t-\frac{\pi}{4}\right), \quad t \in[0,+\infty), \\
u(t)=\psi(t), \quad t \in\left[-\frac{\pi}{4}, 0\right] \\
x(0)=x_{0},
\end{gathered}
$$

where the admissible initial data $\left(x_{0}, \psi\right) \in \mathscr{A}$. Now, we apply Theorem 8 to prove that system (81) is controllable. Let us take

$$
\begin{array}{cc}
\alpha=\frac{1}{2}, \quad E=\left[\begin{array}{lll}
1 & 0 & 0 \\
0 & 0 & 1 \\
0 & 0 & 0
\end{array}\right], \quad A=\left[\begin{array}{lll}
1 & 0 & 0 \\
0 & 1 & 1 \\
1 & 0 & 1
\end{array}\right], \\
B=\left[\begin{array}{ll}
1 & 0 \\
1 & 1 \\
0 & 1
\end{array}\right], \quad C=\left[\begin{array}{ll}
1 & 0 \\
0 & 1 \\
1 & 1
\end{array}\right] .
\end{array}
$$

If we choose $\lambda=1$ such that $\operatorname{det}(\lambda E+A)=2 \neq 0$, then $(E, A)$ is regular. By using the elementary transformation of matrix, one can obtain

$$
\begin{gathered}
\operatorname{rank}[s E-A, B, C]=\operatorname{rank}\left[\begin{array}{ccccccc}
1 & 0 & 1 & \star & \star & \star & \star \\
0 & 1 & -1 & \star & \star & \star & \star \\
0 & 0 & 2 & \star & \star & \star & \star
\end{array}\right]=3, \\
\operatorname{rank}[E, B, C]=\operatorname{rank}\left[\begin{array}{ccccccc}
1 & 0 & 1 & \star & \star & \star & \star \\
0 & 1 & 0 & \star & \star & \star & \star \\
0 & 0 & 1 & \star & \star & \star & \star
\end{array}\right]=3 .
\end{gathered}
$$

Thus, by Theorem 8 , system (81) is controllable.

\section{Conclusions}

In this paper, the reachability and controllability of fractional singular dynamical systems with control delay have 
been investigated. The state structure of fractional singular dynamical systems with control delay has been characterized by analysing the state response and reachable set. A set of sufficient and necessary conditions of controllability criteria for such systems has been established based on the algebraic approach. An example is also presented to illustrate the effectiveness and applicability of the results obtained. Both the proposed criteria and the example show that the controllability property of fractional linear singular dynamical systems is dependent neither on the order of fractional derivative nor on control delay. Comparing with some existing results $[1,23$, 25], we find that the algebraic approach has been extended to consider the controllability of more general fractional singular dynamical systems. Motivated and inspired by the works of Sakthivel et al. [32-35], the approximate controllability of linear (nonlinear) fractional singular dynamical systems will become our future investigative work.

\section{Conflicts of Interests}

The authors declare that there is no conflict of interests regarding the publication of this paper.

\section{Acknowledgments}

The authors are very grateful to the Associate Editor, Professor Han H. Choi, and the anonymous reviewers for their helpful and valuable comments and suggestions which improved the quality of the paper. This work is jointly supported by the National Natural Science Foundation of China under Grant nos. 11071001, 11072059, and 61272530, the Natural Science Foundation of Jiangsu Province of China under Grant no. BK2012741, and the Programs of Educational Commission of Anhui Province of China under Grant nos. KJ2011A197 and KJ2013Z186. This work is also funded by the Deanship of Scientific Research, King Abdulaziz University (KAU), under Grant no. 3-130/1434/HiCi. Therefore, the authors acknowledge technical and financial support of KAU.

\section{References}

[1] L. Dai, Singular Control Systems, vol. 118 of Lecture Notes in Control and Information Sciences, Springer, Berlin, Germany, 1989.

[2] P. Kunkel and V. Mehrmann, Differential-Algebraic Equations: Analysis and Numerical Solution, European Mathematical Society, Zürich, Switzerland, 2006.

[3] E. L. Yip and R. F. Sincovec, "Solvability, controllability, and observability of continuous descriptor systems," IEEE Transactions on Automatic Control, vol. 26, no. 3, pp. 702-707, 1981.

[4] W. S. Tang and G. Q. Li, "The criterion for controllability and observability of singular systems," Acta Automatica Sinica, vol. 21, no. 1, pp. 63-66, 1995.

[5] W. Jiang and W. Z. Song, "Controllability of singular systems with control delay," Automatica, vol. 37, no. 11, pp. 1873-1877, 2001.

[6] S. L. Campbell and V. H. Linh, "Stability criteria for differentialalgebraic equations with multiple delays and their numerical solutions," Applied Mathematics and Computation, vol. 208, no. 2, pp. 397-415, 2009.
[7] H. Wang, A. Xue, and R. Lu, "Absolute stability criteria for a class of nonlinear singular systems with time delay," Nonlinear Analysis: Theory, Methods \& Applications, vol. 70, no. 2, pp. 621630, 2009.

[8] J. Xi, F. Meng, Z. Shi, and Y. Zhong, "Delay-dependent admissible consensualization for singular time-delayed swarm systems," Systems \& Control Letters, vol. 61, no. 11, pp. 1089-1096, 2012.

[9] K. S. Miller and B. Ross, An Introduction to the Fractional Calculus and Fractional Differential Equations, John Wiley \& Sons, New York, NY, USA, 1993.

[10] I. Podlubny, Fractional Differential Equations, vol. 198 of Mathematics in Science and Engineering, Technical University of Kosice, Kosice, Slovakia, 1999.

[11] K. Diethelm, The Analysis of Fractional Differential Equations: An Application-Oriented Exposition Using Differential Operators of Caputo Type, vol. 2004 of Lecture Notes in Mathematics, Springer, Berlin, Germany, 2010.

[12] A. A. Kilbas, H. M. Srivastava, and J. J. Trujillo, Theory and Applications of Fractional Differential Equations, vol. 204 of North-Holland Mathematics Studies, Elsevier Science B.V., Amsterdam, The Netherlands, 2006.

[13] M. Caputo, "Linear model of dissipation whose $Q$ is almost frequency independent II," Geophysical Journal of the Royal Astronomical Society, vol. 13, no. 5, pp. 529-539, 1967.

[14] V. Lakshmikantham and A. S. Vatsala, "Basic theory of fractional differential equations," Nonlinear Analysis: Theory, Methods \& Applications, vol. 69, no. 8, pp. 2677-2682, 2008.

[15] Z. M. Odibat, "Analytic study on linear systems of fractional differential equations," Computers \& Mathematics with Applications, vol. 59, no. 3, pp. 1171-1183, 2010.

[16] Z. X. Zhang and W. Jiang, "Some results of the degenerate fractional differential system with delay," Computers \& Mathematics with Applications, vol. 62, no. 3, pp. 1284-1291, 2011.

[17] K. Sayevand, A. Golbabai, and A. Yildirim, "Analysis of differential equations of fractional order," Applied Mathematical Modelling, vol. 36, no. 9, pp. 4356-4364, 2012.

[18] H. Zhang, J. Cao, and W. Jiang, "General solution of linear fractional neutral differential difference equations," Discrete Dynamics in Nature and Society, vol. 2013, Article ID 489521, 7 pages, 2013.

[19] M. I. Krastanov, "On the constrained small-time controllability of linear systems," Automatica, vol. 44, no. 9, pp. 2370-2374, 2008.

[20] S. Zhao and J. Sun, "A geometric approach for reachability and observability of linear switched impulsive systems," Nonlinear Analysis: Theory, Methods \& Applications, vol. 72, no. 11, pp. 4221-4229, 2010.

[21] R. Manzanilla, L. G. Mármol, and C. J. Vanegas, "On the controllability of a differential equation with delayed and advanced arguments," Abstract and Applied Analysis, vol. 2010, Article ID 307409, 16 pages, 2010.

[22] R. M. Du and C. P. Wang, "Null controllability of a class of systems governed by coupled degenerate equations," Applied Mathematics Letters, vol. 26, no. 1, pp. 113-119, 2013.

[23] T. L. Guo, "Controllability and observability of impulsive fractional linear time-invariant system," Computers \& Mathematics with Applications, vol. 64, no. 10, pp. 3171-3182, 2012.

[24] H. Zhang, J. Cao, and W. Jiang, "Controllability criteria for linear fractional differential systems with state delay and impulses," Journal of Applied Mathematics, vol. 2013, Article ID 146010, 9 pages, 2013. 
[25] W. Jiang, "The controllability of fractional control systems with control delay," Computers \& Mathematics with Applications, vol. 64, no. 10, pp. 3153-3159, 2012.

[26] J. Wang and Y. Zhou, "Analysis of nonlinear fractional control systems in Banach spaces," Nonlinear Analysis: Theory, Methods \& Applications, vol. 74, no. 17, pp. 5929-5942, 2011.

[27] K. Balachandran, J. Kokila, and J. J. Trujillo, "Relative controllability of fractional dynamical systems with multiple delays in control," Computers \& Mathematics with Applications, vol. 64, no. 10, pp. 3037-3045, 2012.

[28] K. Balachandran, J. Y. Park, and J. J. Trujillo, "Controllability of nonlinear fractional dynamical systems," Nonlinear Analysis: Theory, Methods \& Applications, vol. 75, no. 4, pp. 1919-1926, 2012.

[29] K. Balachandran, Y. Zhou, and J. Kokila, "Relative controllability of fractional dynamical systems with delays in control," Communications in Nonlinear Science and Numerical Simulation, vol. 17, no. 9, pp. 3508-3520, 2012.

[30] R. Sakthivel, N. I. Mahmudov, and J. J. Nieto, "Controllability for a class of fractional-order neutral evolution control systems," Applied Mathematics and Computation, vol. 218, no. 20, pp. 10334-10340, 2012.

[31] R. Ganesh, R. Sakthivel, N. I. Mahmudov, and S. M. Anthoni, "Approximate controllability of fractional integrodifferential evolution equations," Journal of Applied Mathematics, vol. 2013, Article ID 291816, 7 pages, 2013.

[32] R. Sakthivel, Y. Ren, and N. I. Mahmudov, "On the approximate controllability of semilinear fractional differential systems," Computers \& Mathematics with Applications, vol. 62, no. 3, pp. 1451-1459, 2011.

[33] R. Sakthivel, R. Ganesh, and S. Suganya, "Approximate controllability of fractional neutral stochastic system with infinite delay," Reports on Mathematical Physics, vol. 70, no. 3, pp. 291311, 2012.

[34] R. Sakthivel and Y. Ren, "Approximate controllability of fractional differential equations with state-dependent delay," Results in Mathematics, vol. 63, no. 3, pp. 949-963, 2013.

[35] R. Sakthivel, R. Ganesh, Y. Ren, and S. M. Anthoni, "Approximate controllability of nonlinear fractional dynamical systems," Communications in Nonlinear Sciences and Numerical Simulation, vol. 18, no. 12, pp. 3498-3508, 2013. 


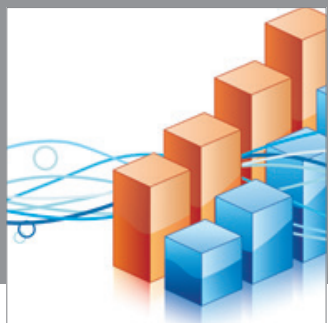

Advances in

Operations Research

mansans

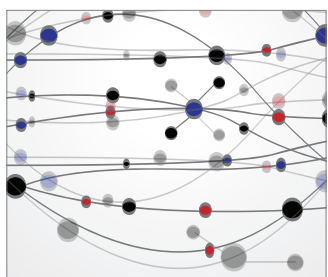

The Scientific World Journal
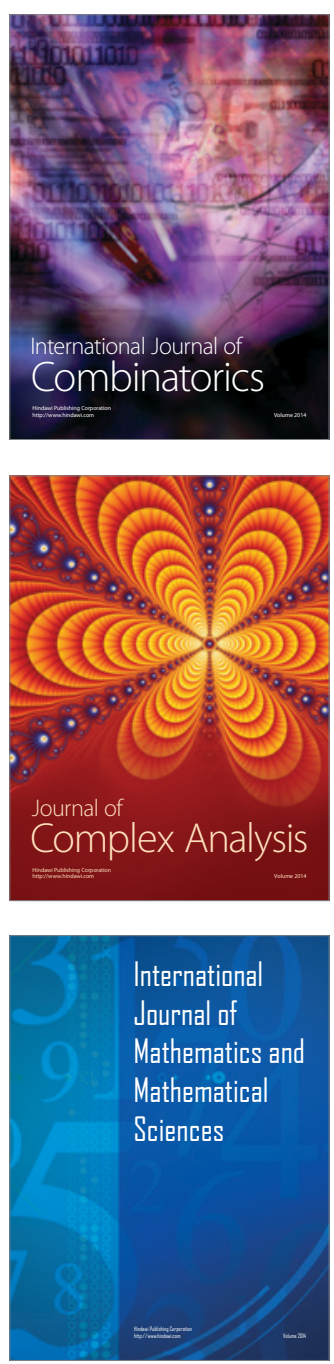
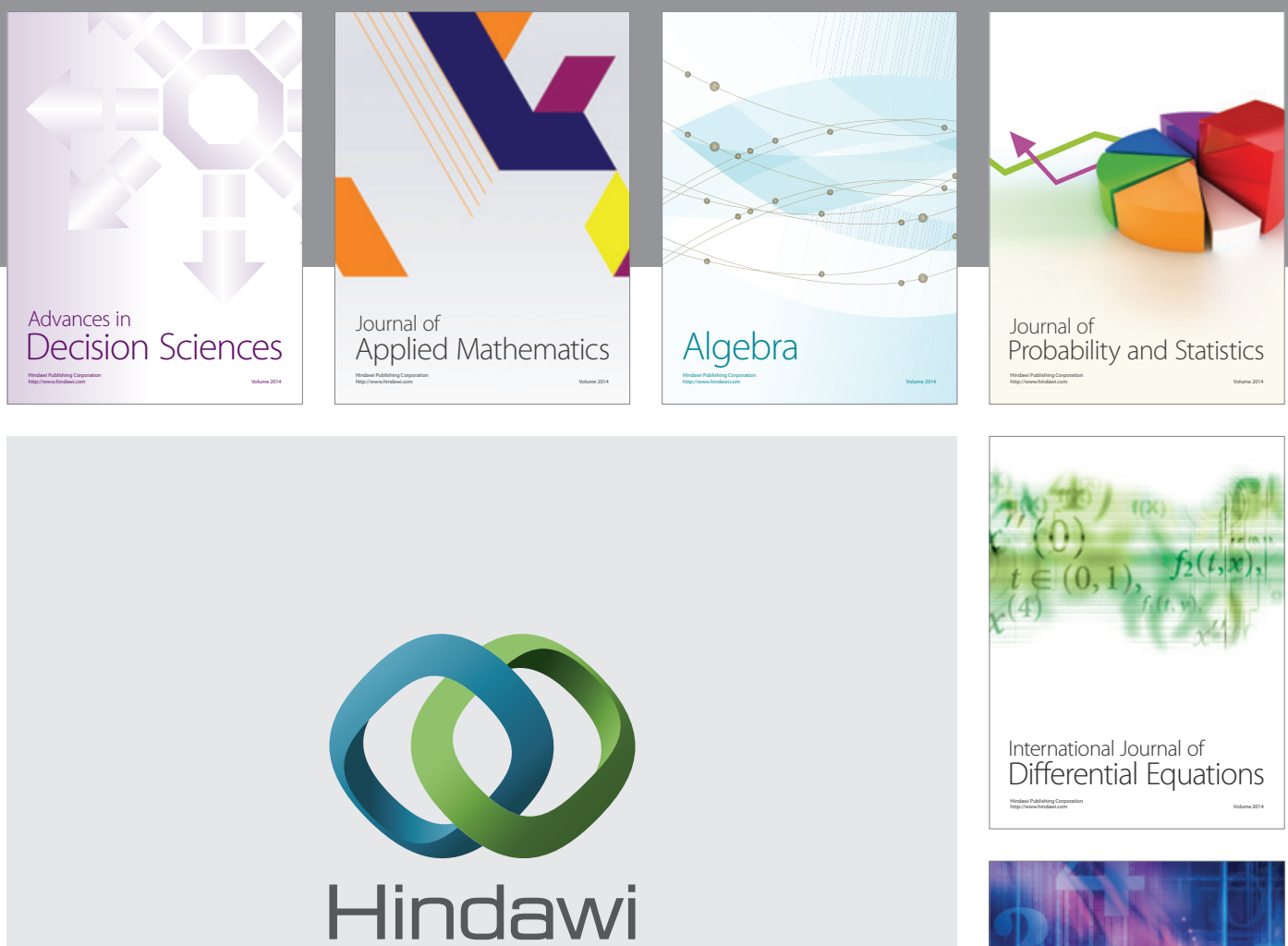

Submit your manuscripts at http://www.hindawi.com
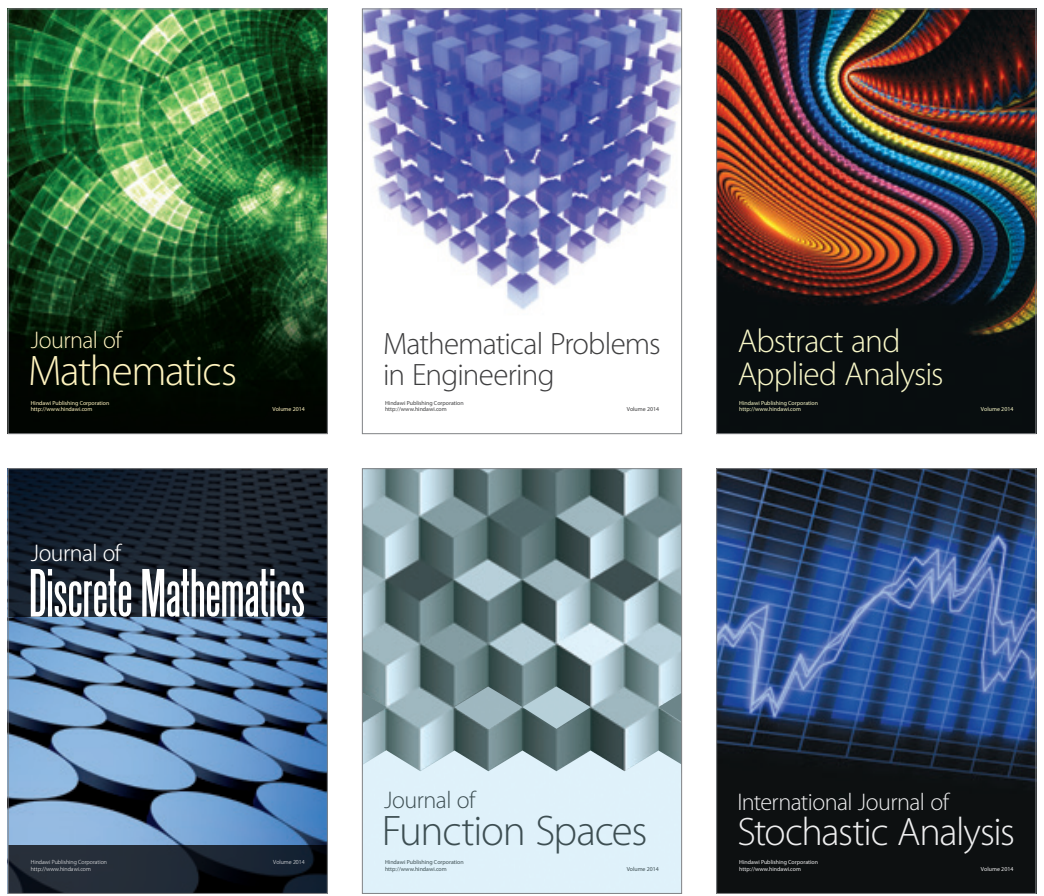

Journal of

Function Spaces

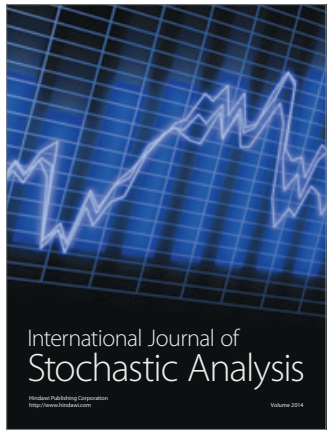

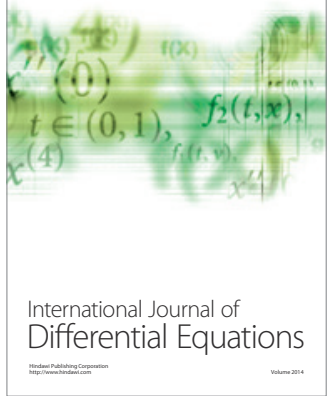
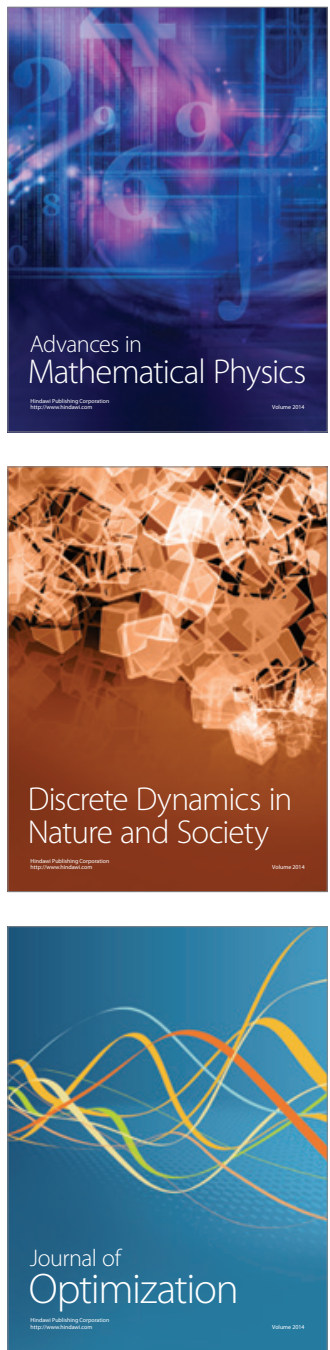